NOTA CIENTÍFICA

\title{
Primer registro de Saguinus fuscicollis melanoleucus (Miranda Ribeiro, 1912) en la Amazonía peruana
}

\author{
First record of Saguinus fuscicollis melanoleucus (Miranda Ribeiro, 1912) \\ in the Peruvian Amazon
José Luis Mena', Alfredo Dosantos², José Grocio Gil², Mario Escobedo², Rolando Aquino $^{4}$ y Jorge Peres ${ }^{5}$

\author{
${ }^{1}$ World Wildlife Fund - Oficina del Programa Perú. Calle Trinidad Morán 853, Lince, Lima 14, Apartado \\ Postal 11-0205. \\ ${ }^{2}$ Facultad de Ciencias Biológicas, Universidad Nacional de la Amazonía Peruana \\ ${ }^{3}$ Parque Nacional Alto Purús, Instituto Nacional de Recursos Naturales \\ ${ }^{4}$ Facultad de Ciencias Biológicas, Universidad Nacional Mayor de San Marcos \\ ${ }^{5}$ Comunidad Amahuaca Santa Rosa, Breu, Ucayali. \\ Email José Mena: JI.Mena@wwfperu.org.pe
}

\section{Resumen}

En el presente trabajo reportamos la presencia de Saguinus fuscicollis melanoleucus en la Amazonía Peruana. Esta subespecie fue registrada, durante los censos de fauna silvestre y también de manera ocasional, en las cuencas altas del río Breu y de la quebrada Beu cerca de la frontera con Brasil en el distrito de Yurúa, departamento de Ucayali. En la Amazonía peruana, los hábitats de Saguinus fuscicollis melanoleucus comprenden el bosque primario y el bosque mixto con Guadua sp. en buen estado de conservación. Esta zona es habitada por las comunidades indígenas Amahuaca, en el lado peruano y Kashinawa, en el lado brasileño.

Palabras claves: Primates, Saguinus fuscicollis melanoleucus, hábitats, distribución, Perú

Presentado: $\quad 06 / 09 / 2006$ Aceptado: $\quad 10 / 11 / 2006$

\section{Abstract}

The fist record of Saguinus fuscicollis melanoleucus to the Peruvian Amazon is reported. This subspecies was registered by wildlife census and by an occasional record from the headwaters of the Breu and Beu rivers near to the limit with Brazil in the district of Yurua, department of Ucayali. The habitat of Saguinus fuscicollis melanoleucus in Peru includes primary forest and forests with Guadua sp. This place is inhabited by Amahuaca (Peruvian side) and Kashinawa (Brazilian side) indigenous communities.

Keywords: Primates, Saguinus fuscicollis melanoleucus, habitats, distribution, Peru.
Saguinus fuscicollis (Spix, 1823) o pichico común es una especie de amplia distribución en los bosques lluviosos de la Amazonía. Ha sido registrado en el extremo sur de Colombia y desde allí hasta el sudeste de Perú, Bolivia y el occidente de Brasil (Aquino y Encarnación, 1994; Eisenberg y Redford, 1999). Se encuentra habitando en bosques primarios y secundarios (Emmons y Feer, 1997), pero también en ambientes modificados por la actividad humana como plantaciones de plátano y en chacras en proceso de maduración (Aquino y Encarnación, 1994; Naughton-Treves et al., 2003). Esta especie fue registrada en varias áreas naturales protegidas del Perú, como los Parques Nacionales de Manú, Otishi, Tingo María, Alto Purús y Yanachaga Chemillén; así como, en la Reserva Nacional Pacaya Samiria, Reservas Comunales Purús y Tamshiyacu-Tahuayo; y en el Santuario Nacional Megantoni (Pacheco et al., 1993; Pacheco y Vivar, 1996; Voss y Emmons, 1996; Aquino y Calle, 2003; Naughton-Treves et al., 2003; Meneses y Aguiar, 2003; Pitman et al., 2003; Vriesendorp et al., 2004).

Saguinus fuscicollis es una especie de tamaño pequeño, que mide un total de $60 \mathrm{~cm}$ y un peso de hasta $550 \mathrm{~g}$ (Emmons y Feer, 1997; Eisenberg y Redford, 1999) Es una especie que convive en grupos de 2 a 12 individuos, es típicamente omnívoro y se alimenta principalmente de frutos, insectos, resinas, néctar y pequeños vertebrados (Aquino y Encarnación, 1994; Emmons y
Feer, 1997; Heymann et al., 2000; Knogge y Heymann, 2006). En su medio natural suele encontrarse en simpatría con otras especies de su género como $S$. mystax, S. labiatus o $S$. imperator, con quienes muchas veces se asocian para formar grupos mixtos (Aquino y Encarnación, 1994; Tirado-Herrera y Heymann, 2004), inclusive con Callimico goeldii (Porter, 2001). En el Perú, es común observar a $S$. fuscicollis formando grupos mixtos de forrajeo con $S$. imperator, tales asociaciones son de largo plazo, y aparentemente proveerían cierta ventaja en la defensa del área de forrajeo (Heymann, 1990; Eisenberg y Redford, 1999).

Rylands et al. (2000), reconocen para S. fuscicollis un total de 33 subespecies, las mismas que presentan diferencias morfológicas que se deben generalmente a diferencias genéticas antes que a diferencias ambientales (Kohn et al., 2001). Aquino y Encarnación (1994) reportaron un total de seis subespecies para la Amazonía peruana: $S$. $f$. fuscicollis, $S$. $f$. leucogenys, $S$. $f$. illigeri, $S$. f. lagonotus, $S$. f. nigrifons, y $S$. f. weddelli. De acuerdo al trabajo de Aquino y Encarnación, en el departamento de Ucayali ocurriría S. f. weddelli. Asimismo, Hershkovitz (1977) señaló previamente que el área que se encuentra entre las cuencas de los ríos Purús y Juruá no fue explorada convenientemente.

En julio del 2005 durante una evaluación biológica en la desembocadura de la quebrada Beu, ubicada en el distrito 


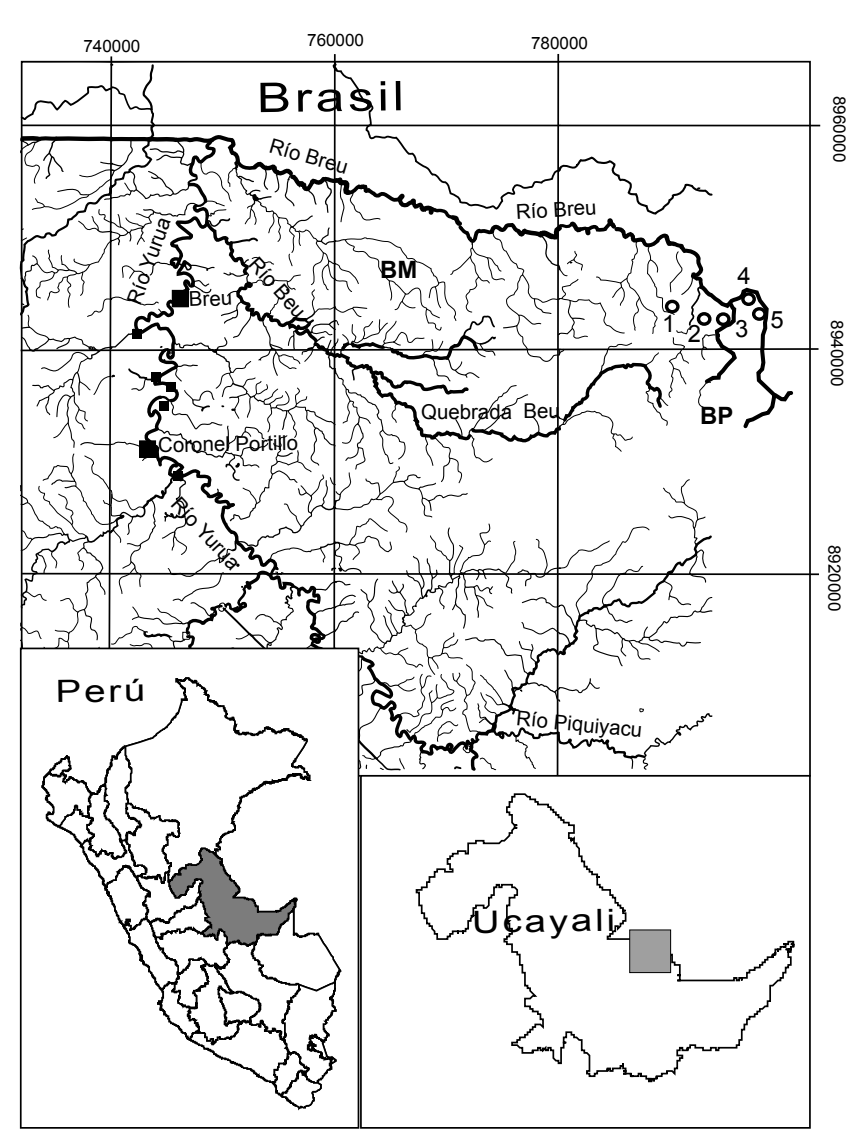

Figura 1. Sitios en donde se registró la presencia de grupos de Saguinus fuscicollis melanoleucus en la Amazonía peruana. $\mathrm{BP}=$ Bosque primario, $\mathrm{BM}=$ Bosque mixto con paca. Sitios de avistamiento: 1, 2, 3, 4, 5 (ver Tabla 1).

de Yurúa en el departamento de Ucayali (ver Figura 1), los pobladores locales de la etnia Amahuaca nos comentaron de la presencia de un "pichico blanco" que habitaba en las cabeceras de esta quebrada. A través de encuestas realizadas a los cazadores Amahuaca descubrimos que esta especie era bastante común en la zona, sin embargo, en aquella oportunidad no se logró observar individuo alguno que permita una determinación correcta de la especie de "pichico" o "Shipi" (nombre Amahuaca de las especies del género Saguinus).

En marzo del 2006, durante una segunda evaluación biológica, llevada a cabo en la cuenca del río Breu, afluente del río Yurúa (más conocido como Juruá en territorio brasileño) se registraron
Tabla 1. Registros de Saguinus fuscicollis melanoleucus en la Amazonía de Perú y Brasil.

\begin{tabular}{|c|c|c|c|}
\hline Localidad & Longitud & Latitud & Fuente \\
\hline Río Envira, Acre ${ }^{1}$ & ¿? & ¿? & Tagliaro et al. 2005 \\
\hline Río Eirú ${ }^{1 *}$ & $54^{\circ} 16^{\prime} 1^{\prime \prime}$ & $6^{\circ} 40^{\prime} 59^{\prime \prime}$ & Hershkovitz (1977) \\
\hline Alto Juruáít & ¿? & ¿? & Peres (1993) \\
\hline Río Juruá ${ }^{1^{*}}$ & ¿? & ¿? & Hershkovitz (1977) \\
\hline Sitio $^{1,2^{*}}$ & $72^{\circ} 21^{\prime} 34^{\prime \prime}$ & $9^{\circ} 32^{\prime} 49^{\prime \prime}$ & Transecto lineal \\
\hline Sitio $^{2,2^{*}}$ & $72^{\circ} 19^{\prime} 59^{\prime \prime}$ & $9^{\circ} 33^{\prime} 21^{\prime \prime}$ & Transecto lineal \\
\hline Sitio $^{3,2^{*}}$ & $72^{\circ} 19^{\prime} 03^{\prime \prime}$ & $9^{\circ} 33^{\prime} 29^{\prime \prime}$ & Registro ocasional \\
\hline Sitio $^{4,2^{*}}$ & $72^{\circ} 17^{\prime} 58^{\prime \prime}$ & $9^{\circ} 32^{\prime} 02^{\prime \prime}$ & Transecto lineal \\
\hline Sitio $^{5,2^{*}}$ & $72^{\circ} 16^{\prime} 58^{\prime \prime}$ & $9^{\circ} 32^{\prime} 28^{\prime \prime}$ & Transecto lineal \\
\hline
\end{tabular}

${ }^{1 *}$ Brasil

$2^{\star}$ Perú

$1,2,3,4,5$ Sitios en donde se registró la presencia de grupos de Saguinus fuscicollis melanoleucus en la Amazonía peruana, ver figura 1.

varios grupos de Saguinus $f$. melanoleucus en asociación con $S$. imperator (ver Figura 1). Los habitantes indígenas Kashinawa asentados a lo largo del río Breu reconocen a estos primates comúnmente como "Hushuxipi". En esta oportunidad, los registros de esta subespecie correspondieron a dos fuentes: un registro ocasional de un grupo observado por un equipo de ictiólogos durante sus evaluaciones de campo a orillas del río Breu (en territorio peruano), y el segundo registro de cuatro grupos durante los censos de fauna silvestre realizados en un bosque mixto con paca (Guadua sp.) y en un bosque primario (ver Figura 1). El bosque primario estaba representado principalmente por representantes de las familias Fabaceae, Magnoliopsidae, Violaceae, Moraceae, Arecaceae, Bombacaeae y Meliaceae; mientras que el bosque mixto principalmente por Magnoliopsidae, Moraceae, Bombacaceae, Fabaceae, Rubiaceae, Arecaceae, Poaceae y Euphorbiaceae. Los censos fueron realizados en seis transectos, cada uno de $5 \mathrm{~km}$ cada uno, los cuales iniciaban a 200 o $300 \mathrm{~m}$ desde la orilla del río a fin de evitar el uso con fines de caza. Los censos se realizaron desde las 07:45 a 16:00 h (hora local).

Los individuos observados presentaron el patrón de coloración del pelaje que Hershkovitz (1977) señaló como característico de $S$. f. acrensis Carvalho 1957, es decir, la cabeza, el manto y los hombros ligeramente blancos salpicado de ante o amarillo; la piel del rostro y orejas de color negro, finamente cubierto con pelos blancos cortos; la espalda media e inferior veteada monótonamente de beige ocráceo; ancas y muslos anaranjado;

Figura 2. Ejemplar de Saguinus fuscicollis melanoleucus, cazado por un poblador amahuaca en la cuenca alta del río Breu en territorio peruano.

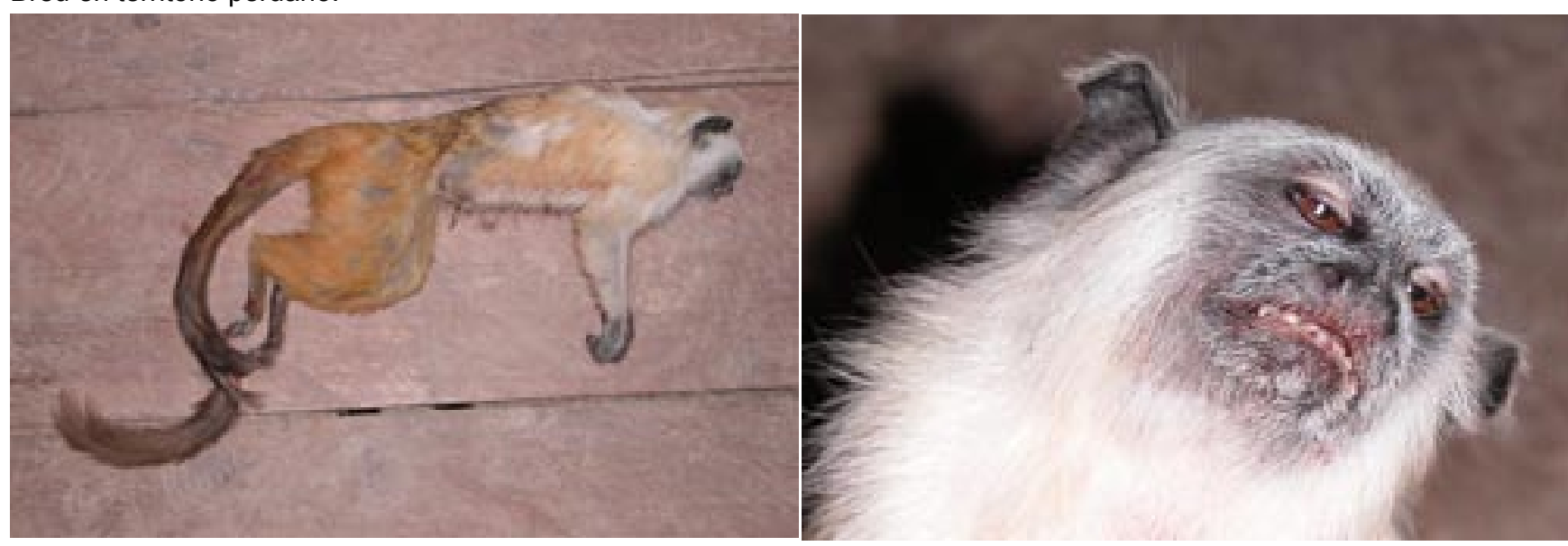


vientre amarillento; lado externo de las manos blancuzcas y cola anaranjado-amarillenta, pero más oscuro en el ejemplar colectado en Breu (ver Figura 2). Hershkovitz mencionó que la localidad tipo de $S$. f. acrensis es Pedra preta, localizada a $1,5 \mathrm{~km}$ de Vila Taumaturgo, en la margen derecha del río Juruá, Acre en Brasil, cerca de la frontera con Perú. Es importante señalar que CoimbraFilho (1990) listó a S. f. melanoleucus, S. f. acrensis y S. f. crandalli como subespecies de $S$. melanoleucus. Asimismo, según el estudio realizado por Peres (1993)en el río alto Juruá, $S$. f. acrensis no es considerado como una forma valida sino un híbrido entre $S$. $f$. fuscicollis y $S$. $f$. melanoleucus. Rylands et al.(2000) incluye a $S$. $f$. melanoleucus, como una subespecie válida de $S$. fuscicollis, sin embargo, Tagliaro et al. (2005), basados en estudios moleculares usando $\mathrm{ADN}$ mitocondrial, argumentan que $S$. $f$. melanoleucus y $S$. $f$. weddelli no alcanzan a tener un nivel de divergencia tal que permita apoyar la división de estos morfotipos en subespecies. No obstante, es importante destacar que Wilson y Reeder (2005)reconocen el nivel específico de Saguinus melanoleucus.

Durante la evaluación de campo, en la única oportunidad de manipular un ejemplar de $S$. $f$. melanoleucus cazado por un poblador amahuaca, registramos las siguientes medidas: longitud del cuerpo de $205 \mathrm{~mm}$, longitud de la cola de $300 \mathrm{~mm}$, longitud de pata de $65 \mathrm{~mm}$ y longitud de la oreja de $29 \mathrm{~mm}$. El cráneo del espécimen fue depositado en la colección de mamíferos del Museo de Historia Natural de la Universidad Nacional Mayor de San Marcos.

Saguinusf. melanoleucus en la Amazonía peruana es simpátrica con otras especies de primates, tal y como fue observado durante los censos por transecto de $130 \mathrm{~km}$ Entre estos podemos mencionar a Saguinus imperator, Pithecia monachus, Alouatta seniculus y Cebus apella. A través de encuestas también fue registrada la presencia de Ateles chamek, Callicebus cupreus y Aotus sp. Las abundancias relativas por $100 \mathrm{Km}$ de recorrido para las especies de $S$. f. melanoleucus y $S$. imperator en la cuenca alta del río Breu son 24,62 y 22,31 individuos, respectivamente.

En el Brazil, S. f. melanoleucus fue reportado anteriormente en el oeste del estado de Amazonas, entre los ríos Juruá y Tarauacá (Hershkovitz, 1977). Esta nota reporta el registro más occidental para esta subespecie (Ver Tabla 1), adicionando así una subespecie (S.f. melanoleucus) más de S. fuscicollis para el Perú y para el departamento de Ucayali (con la reserva de que si en adelante se confirma su nivel específico, entonces representaría el primer registro de $S$. melanoleucus para Perú). El área de distribución de esta subespecie en el lado peruano comprende un paisaje de bosque tropical lluvioso primario y bosque mixto con Guadua sp. "paca" cerca de la frontera con Brasil, correspondiente a la cuenca altas del río Breu y el curso superior de la quebrada Beu (según señalan los pobladores locales); ambas en buen estado de conservación (ver Figura 1). En esta parte del territorio peruano sólo se practica la cacería de subsistencia y considerando que las poblaciones humanas asentadas en esta cuenca son bastante reducidas, el impacto sobre la fauna silvestre no parece ser significativo, más aún, porque las comunidades indígenas de la zona han mostrado un gran interés por la conservación de sus recursos, y si a ella adicionamos a esta pequeńa especie de primate como un componente sin importancia en la dieta local, nos permite inferir que su conservación podría estar asegurada (siempre y cuando no haya un cambio drástico en el uso de los recursos).
No obstante, más investigaciones serán necesarias para conocer con precisión su rango de distribución en el lado peruano. Esta nota también demuestra lo importante que es reconocer y valorar el conocimiento de los pueblos indígenas sobre la fauna, que en este caso nos permitió reportar una nueva subespecie de Saguinus fuscicollis para el Perú.

\section{Agradecimientos}

Nuestra profunda gratitud a la comunidad Amahuaca de Santa Rosa que gentilmente nos comentó la existencia del "pichico blanco". Nuestro agradecimiento a la organización indígena ACONADYISH que nos facilitó el trabajo en el área de estudio $\mathrm{y}$ al INRENA por los permisos respectivos para las evaluaciones de campo. A todos los amigos amahuacas, yaminahuas y asheninkas peruanos, así como a los Kashinahuas brasileños que nos brindaron su amistad y apoyo durante las evaluaciones. Este estudio se desarrolló dentro del proyecto "Iniciativa para la protección de cabeceras Amazónicas II", financiado por la Gordon and Betty Moore foundation y ejecutado por la World Wildlife Fund-Oficina del programa Perú. Blanca Rengifo y Vanessa Chipollini observaron el primer grupo de $S$. $f$. melanoleucus durante sus evaluaciones de recursos hidrobiológicos.

\section{Literatura citada}

Aquino, R. y A. Calle. 2003. Evaluación del estado de conservación de los mamíferos de caza: un modelo comparativo en comunidades de la Reserva Nacional Pacaya Samiria (Loreto, Peru). Revista Peruana de Biología 10:163-174.

Aquino, R. y F. Encarnación. 1994. Los primates del Perú. Primate Report 40:43-127.

Coimbra-Filho, A. F. 1990. Sistemática, distribuição geográfica e situação atual dos símios brasileiros (Platyrrhini-Primates). Revista Brasileira de Biologia 50:1063-1079.

Eisenberg, J. F. y K. H. Redford. 1999. Mammals of the Neotropics. The Central Neotropics. Ecuador, Peru, Bolivia, Brazil. The University of Chicago Press.

Emmons, L. H. y F. Feer. 1997. Neotropical Rainforest Mammals, 2nd edn. The University of Chicago Press, Chicago.

Hershkovitz, P. 1977. Living new world monkeys (Platyrrhini): with an introduction to primates. The University of Chicago Press, Chicago \& Londres.

Heymann, E. W. 1990. Interspecific relations in a mixed-species troop of moustached tamarins, Saguinus mystax, and saddle-back tamarins, Saguinus fuscicollis (Platyrrhini: Callitrichidae), at the Rio Blanco, Peruvian Amazonia. American Journal of Primatology 21:115-127.

Heymann, E. W., C. Knogge y E. R. Tirado Herrera. 2000. Vertebrate predation by sympatric tamarins, Saguinus mystax and Saguinus fuscicollis. American Journal of Primatology 51:153-158.

Knogge, C. y E. W. Heymann. 2006. Seed dispersal by simpatric tamarins, Saguinus mystax and Saguinus fuscicollis: diversity and characteristics of plant species. Folia Primatologica 74:33-47.

Kohn, L. A., L. B. Langton y J. M. Cheverud. 2001. Subspecific genetic differences in the saddle-back tamarin (Saguinus fuscicollis) postcranial skeleton. American Journal of Primatology 54:41-56.

Meneses, E. y J. M. Aguiar. 2003. Three new natural protected areas in Peru. Neotropical Primates 11:122-124.

Naughton-Treves, L., J. L. Mena, A. Treves, N. Alvarez y V. C. Radeloff. 2003. Wildlife Survival Beyond Park Boundaries: the Impact of Slash-and-Burn Agriculture and Hunting on Mammals in Tambopata, Peru. Conservation Biology 17:1106-1117. 
Pacheco, V., B. D. Patterson, J. L. Patton, L. H. Emmons, S. Solari y C. F. Ascorra. 1993. List of mammal species known to occur in Manu Biosphere Reserve, Peru. Publ.Mus.Hist. Nat.UNMSM (A) 44:1-12.

Pacheco, V. y E. Vivar. 1996. Annotated checklist of the non-flying mammals at Pakitza, Manu Reserve Zone, Manu National Park. In: D. E. Wilson and A. Sandoval, eds. Manu: The Biodiversity of Southeastern Peru. Smithsonian Institution, Whasington, D. C. Pages 577-625

Peres, C. A. 1993. Notes on the primates of the Juruá river, western Brazilian Amazonia. Folia Primatologica 61:97-103.

Pitman, R. L., H. Beck, y P. M. Velazco. 2003. Mamíferos terrestres y arbóreos de la selva baja de la Amazonía Peruana; entre los ríos Manu y Alto Purús. In: R. L. Pitman, N. Pitman and P. Álvarez, eds. Alto Purus: Biodiversidad, Conservación y Manejo. Center for Tropical Conservation, Nicholas school of the environment, Duke University, Lima. Pages $109-122$

Porter, L. M. 2001. Benefits of polyspecific associations for the Goeldi's monkey (Callimico goeldii). American Journal of Primatology 54:143-158.
Rylands, A. B., H. Schneider, A. Langguth, R. A. Mittermeier, C. P. Groves y E. Rodríguez-Luna. 2000. An assessment of the diversity of new world primates. Neotropical Primates 8:61-92.

Tagliaro, C. H., H. Schneider, I. Sampaio, M. P. C. Schneider, M. Vallinoto y M. Stanhope. 2005. Molecular phylogeny of the genus Saguinus (Platyrrhini, Primates) based on the ND1 mitochondrial gene and implications for conservation. Genetics and Molecular Biology 28:46-53.

Tirado-Herrera, E. R. y E. W. Heymann. 2004. Behavioural changes in response to an injured group member in a group of wild moustached tamarins (Saguinus mystax). Neotropical Primates 12:13-15.

Voss, R. S. y L. H. Emmons. 1996. Mammalian diversity in Neotropical lowland rainforests: A preliminary assessment. Bulletin of the American Museum of Natural History 230:1-115.

Vriesendorp, C., L. Rivera-Chávez, D. Moskovits, y J. Shopland. 2004. Perú: Megantoni. Rapid Biological Inventories Report 15. The Field Museum, Chicago, Illinois.

Wilson, D. E. y D. M. Reeder. 2005. Mammal species of the world, 3 edn. Johns Hopkins University Press. 